\title{
Factors associated with referral compliance of abnormal immunochemical faecal occult blood test
}

\author{
Chia-Ming Li, Ming-Neng Shiu, Shu-Li Chia, Jen-Pei Liu, Tony Hsiu-Hsi Chen and Wei-Chu Chie

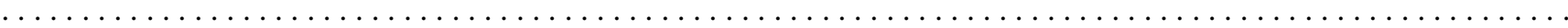

J Med Screen 2007; 14:186-190

See end of article for authors' affiliations

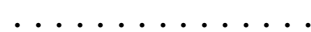

Correspondence to: Wei-Chu Chie, Room 520, 17 Xuzhou Road, Taipei 10055, Taiwan;

weichu@ntu.edu.tw

Accepted for publication 8 July 2007

\begin{abstract}
Objective This study investigated factors associated with the referral compliance of positive immunochemical faecal occult blood test (iFOBT).

Setting Data were from a subset of people who received iFOBT at Taipei county of Taiwan in 2005. Methods All subjects with positive iFOBT were referred to hospital for further diagnostic examinations. In total, 226 such subjects who did not accept referral within 60 days were identified as the non-compliant group from the record of Public Health Bureau. Frequency-matched 219 subjects were sampled from the 599 people who accepted referral within 60 days as the compliant group. Telephone interviews were performed according to questionnaire designed basically under the Health Belief Model. Multiple logistic regression was used to assess effects of possible associated factors for referral compliance.

Results A total of 145 persons in the compliant group and 115 persons in the non-compliant group completed the interview. Factors including 'perceived susceptibility' and 'cue to action: information' were positively associated with, while 'casual personality' was negatively associated with referral compliance.
\end{abstract}

Conclusions Three factors in Health Belief Model were associated with referral compliance after positive FOBT.

\section{INTRODUCTION}

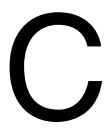

olorectal cancer is the third in cancer incidence ${ }^{1}$ and the fourth in cancer mortality ${ }^{2}$ in Taiwan. Mortality can be reduced by using faecal occult blood test $(\mathrm{FOBT})^{3,4}$ and complete diagnostic examination (CDE) after positive FOBT. ${ }^{5-8}$ The US preventive taskforce suggested annual FOBT examination for colon cancer screening. ${ }^{9}$ People aged between 50 and 69 years can receive free immunochemical FOBT (iFOBT) every two years in Taiwan.

People with positive result in FOBT should be followed with CDE to reduce the mortality of colorectal cancer. ${ }^{5-8}$ However, most previous researches focused on factors affecting the compliance of receiving first-line screening tests. The aim of this study was to investigate factors associated with the referral compliance of people with positive iFOBT result in Taipei County of Taiwan.

\section{METHODS}

\section{Model, definition of variables and questionnaire design}

We defined the variables and designed our questionnaire basically according to the Health Belief $\operatorname{Model}^{10}$ (Appendix 1).

The dependent variable was 'compliance with referral to recommended hospital for further diagnostic examination within 60 days' (compliance with referral) according to Baig et al. ${ }^{11}$ and the administrative capacity of the Public Health Bureau of Taipei County Government. Predictive factors according to Health Belief Model and related studies included: (i) individual perception: perceived susceptibility, ${ }^{12}$ severity of disease $\mathrm{e}^{13-16}$ and perceived health status, ${ }^{17}$ (ii) modifying factors: personality, ${ }^{18}$ knowledge $^{19,20}$ and cues to action, ${ }^{12,13,15,19-22}$ (iii) likelihood of action: perceived benefit $t^{22}$ and barrier to behaviour change $\mathrm{e}^{17,19,22}$ and (iv) personal health practice: fixed health provider $^{23}$ (doctor) and personal health behaviour. ${ }^{17,22}$

\section{Questionnaire testing and revision}

The preliminary questionnaire was sent to five experts for content validity. The questionnaire was revised according to their comments. Six persons with positive iFOBT in Taipei City were selected to do the pre-test before starting the investigation. Questions that cannot be fully understood were further revised (Appendix 2).

\section{Subjects and sample size estimation}

According to the results of a previous study in southern Taiwan, ${ }^{20}$ if we assumed a proportion of 0.2 as a factor in the non-compliant group, we need 95 subjects in each group for an equal-size design to test an odds ratio (OR) of 2.5 in a two-sided test at a significant level of 0.05 and power of 0.80. In Taipei County, a total of 38,504 people received iFOBT and $874(2.3 \%)$ had positive result in 2005 . Twentyone subjects whose age was not surely between 50 and 69 years and 28 subjects whose follow-up duration was unknown were excluded. A total of 599 subjects who received referral within 60 days after positive iFOBT were classified as the compliant group. Another 124 subjects who did not accept referral and 102 subjects who did not accept referral within 60 days were classified as the non-compliant group. Flow diagram of study subjects is shown in Figure 1. We sampled 219 subjects as the compliant group by an 

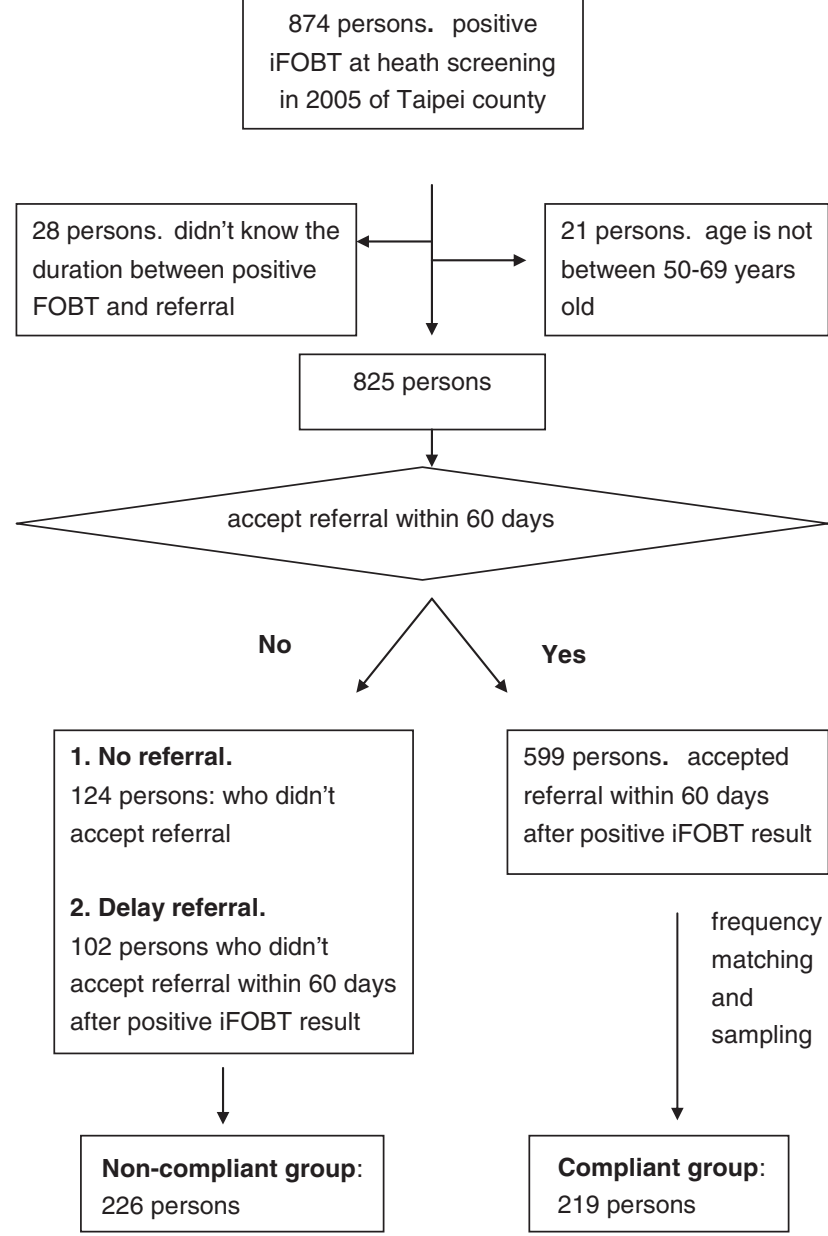

Figure 1 Flow diagram of study subjects

age-gender frequency-matched sampling from the 599 compliant subjects.

\section{Ethical review, study procedure, personal data protection}

This study was approved by the Institutional Review Board of the College of Public Health of National Taiwan University. Two trained interviewers conducted telephone survey from February to March 2006. Participants' oral consents were obtained and witnessed by interviewers. Workers joining in telephone interview and data management signed declaration of confidentiality.

\section{Data processing and statistical methods}

For subjects who completed over $80 \%$ of questions, the missing data and answers of 'Unknown, I don't know' or 'it depends' were recorded as a neutral answer except for special indications. Cronbach's $\alpha$ coefficient was used to assess the internal consistency of questions in each factor (whether the scores of these questions were interrelated). Answers of questions from each factor of Health behaviour Model were summed up as a factor score. Because the distributions of scores of the scales were mostly not normal, we performed tri- or bisection for each score into high, middle and low subgroups according to the distribution in the non-compliant group (reference group).
Simple logistic regression was used to estimate the OR of each factor predicting the compliance to the referral in time. Significant factors in simple logistic regression were gathered in multiple logistic regression model to examine the relative contribution of factors associated with compliance. All hypothesis testing was conducted assuming a 0.05 significant level and a two-sided alternative hypothesis.

We also examined the consistency of positive family history of colon cancer or polyp between the questionnaire and the record of Public Health Bureau (95.2\% consistent), and the consistency of accepting referral between questionnaire and medical records ( $100 \%$ consistent) for a small sample of 21 patients.

\section{RESULTS}

\section{Response rate}

The response rate of complete telephone interview was $55.0 \%(115 / 209)$ in the non-compliant group and $61.4 \%$ $(145 / 236)$ in the compliant group. There was no significant difference in age $(P=0.17)$ and gender $(P=0.66)$ between respondents and non-respondents.

\section{Internal consistency of factors}

The Cronbach's $\alpha$ coefficients of Health Belief Model factors in this study varied from perceived susceptibility $(0.34)$ to perceived benefit (0.83). Only one was poor (perceived susceptibility, 0.34). Two were good (perceived benefit, 0.83 , and barrier to behaviour change, 0.80). Others were fair (between 0.4 and 0.7).

\section{Effects of different factors on referral compliance}

There was no difference in age, gender, education level, marital status and employment status between two groups (Table 1). In univariate analysis, people with higher perceived susceptibility score, lower casual personality score, higher family worry score and higher information score were associated with referral compliance after positive iFOBT (Table 2). In the final force-in model of multiple logistic regression, susceptibility, casual personality and information score remained associated with referral compliance. The OR for medium versus low susceptibility score $=2.11$, 95\% confidence interval (CI): 1.06-4.20, $P=0.03$. The $\mathrm{OR}$ for medium versus high casual personality score $=3.46,95 \%$ CI: $1.09-10.96, P=0.03$. The OR for high versus low information score $=1.99,95 \%$ CI: $1.05-3.77$, $P=0.04$ (Table 2).

\section{DISCUSSION}

This study revealed that 'perceived susceptibility' was one of the significant factors associated with referral compliance, consistent with the findings of Shield's ${ }^{12}$ study. We also found that 'casual personality' was inversely associated with referral compliance. Pulkki et al. ${ }^{18}$ also suggested personality (type A personality) might be a potential contributor to socioeconomic differences in health behaviour. 'Cue to action - information' was another significant factor in this study, consistent with the findings of previous studies. ${ }^{12,13,15,19-22}$ Marcus et al. ${ }^{13}$ and Lerman et al. ${ }^{19}$ found that providing personalized information can enhance follow-up compliance. In detailed analysis (not shown here), encouragement from relatives, friends and doctors was found more important. Chinese usually cherish the 
families' opinion. Encouragement from family can play a good role in practice in Taiwan.

'Perceived benefit' was not associated with referral compliance in this study. It is possible that we would have conducted this study about six months to one year after they knew the result of screening. Most of the subjects in either group did not have colorectal cancer. This experience might reduce their belief in the benefits of CDE. 'Barrier' was also found not associated with referral compliance in this study. This may be the result of health centre staffs' efforts to reduce the barrier of referral, especially in those who refused the referral (the non-compliant group) or the true experience of uncomfortable colonoscopy after referral. 'Knowledge' was not associated with referral compliance, unlike previous studies. ${ }^{19,20}$ The reason is that knowledge of both groups was equally inadequate; less than half of the interviewees knew the purpose of iFOBT, only around 30\% knew the medical procedure after positive iFOBT and less than $30 \%$ knew positive iFOBT might be due to colorectal polyp or cancer.

Table 1 Demographic data of two groups

\begin{tabular}{llll}
\hline & $\begin{array}{l}\text { Compliant } \\
\text { group }\end{array}$ & $\begin{array}{l}\text { Non-compliant } \\
\text { group }\end{array}$ & $P$ value \\
\hline Age (years) & $59.7 \pm 5.7$ & $59.6 \pm 6.4$ & $0.73^{*}$ \\
\hline & No. (\%) & No. (\%) & \\
\hline $\begin{array}{l}\text { Education level } \\
\text { Below elementary school }\end{array}$ & $87(62.6)$ & $62(58.5)$ & $0.51^{\dagger}$ \\
Above junior high school & $52(37.4)$ & $44(41.5)$ & \\
Gender & & & \\
Men & $79(54.5)$ & $74(64.4)$ & $0.11^{\dagger}$ \\
Women & $66(45.5)$ & $41(35.7)$ & \\
Marital status & & & \\
Married & $144(98.6)$ & $114(98.3)$ & $0.87^{\dagger}$ \\
Others & $2(1.4)$ & $2(1.7)$ & \\
$\begin{array}{l}\text { Occupation } \\
\text { Without job }\end{array}$ & $104(71.7)$ & $81(70.4)$ & $0.82^{\dagger}$ \\
With job & $41(28.3)$ & $34(29.6)$ & \\
\hline${ }^{*}$-test & & & \\
${ }^{2} x^{2}$ test & & &
\end{tabular}

This study had following limitations. The original population who attended iFOBT was self-selected. The records of Public Health Bureau could not guarantee that the subjects had recommended diagnostic examinations such as colonoscopy. Besides, some factors had low Cronbach's $\alpha$ coefficient. It is possible that questions in these factors represent different concepts. For example, both rectal bleeding and abdominal discomfort in 'cue to action: symptom' might have measured different problems of the subjects. Furthermore, in the non-compliant group, there is a small group (112 subjects) of delayed referral (more than 60 days) subjects whose characteristics were similar to that of the compliant group. However, only 45 subjects completed the interview, the number of subjects was not enough for further classification in this study. The interview was conducted 4-11 months after positive iFOBT. Compliant subjects may have better access to related information or better memory to factors supposed to be associated during the process of CDE. On the other hand, the non-compliant subjects might have received more information from health centre staff to improve their compliance. Differences in responses rates between the two groups may be a source of bias. History effect might also exist. Furthermore, the questionnaire was designed under the Health Belief Model and there may be other possible factors associated with referral compliance. Finally, the subjects were from Taipei County. The results may be different if the study was conducted in a different district.

\section{CONCLUSIONS}

This study helped to identify the factors associated with compliance to referral of abnormal iFOBT. People with higher perceived susceptibility score, lower casual personality score and higher information score were associated with referral compliance after positive iFOBT.

\section{ACKNOWLEDGEMENTS}

This study was supported by a grant from the National Science Council, Taiwan, NSC 94-2314-B-002-098. The authors also thank the administrative help of Public

Table 2 Crude and adjusted odds ratios (ORs) of factors associated with referral compliance

\begin{tabular}{|c|c|c|c|c|c|c|}
\hline \multirow[b]{2}{*}{ Factors } & \multirow{2}{*}{$\begin{array}{l}\text { Compliant group } \\
(n=145)(\%)\end{array}$} & \multirow{2}{*}{$\begin{array}{l}\text { Non-compliant group } \\
(n=115)(\%)\end{array}$} & \multicolumn{2}{|l|}{ Crude odds ratio } & \multicolumn{2}{|l|}{ Adjusted odds ratio ${ }^{\dagger}$} \\
\hline & & & OR $(95 \% \mathrm{Cl})$ & $P$ value & OR $(95 \% \mathrm{CI})$ & $P$ value \\
\hline $\begin{array}{l}\text { Susceptibili } \\
\text { High } \\
\text { Medium } \\
\text { Low }\end{array}$ & $\begin{array}{l}60(41.4) \\
57(39.3) \\
28(19.3)\end{array}$ & $\begin{array}{l}43(37.4) \\
35(30.4) \\
37(32.2)\end{array}$ & $\begin{array}{l}2.49(1.33-4.67) \\
2.50(1.33-4.72) \\
1 \text { (referent) }\end{array}$ & $\begin{array}{r}<0.01^{* *} \\
0.01^{* *}\end{array}$ & $\begin{array}{l}1.93(0.98-3.84) \\
2.11(1.06-4.20) \\
1 \text { (referent) }\end{array}$ & $\begin{array}{l}0.06 \\
0.03^{*}\end{array}$ \\
\hline $\begin{array}{l}\text { Casual pers } \\
\text { Low } \\
\text { Medium } \\
\text { High }\end{array}$ & $\begin{aligned} 78 & (54.8) \\
62 & (43.8) \\
5 & (3.4)\end{aligned}$ & $\begin{array}{l}58(50.4) \\
41(35.7) \\
16(13.9)\end{array}$ & $\begin{array}{l}4.30 \text { (1.49-12.42) } \\
4.84 \text { (1.65-14.23) } \\
1 \text { (referent) }\end{array}$ & $\begin{array}{l}0.01 * * \\
<0.001 * *\end{array}$ & $\begin{array}{l}2.41(0.75-7.71) \\
3.46(1.09-10.96) \\
1 \text { (referent) }\end{array}$ & $\begin{array}{l}0.14 \\
0.03^{*}\end{array}$ \\
\hline $\begin{array}{l}\text { Family worr } \\
\text { High } \\
\text { Low }\end{array}$ & $\begin{aligned} 131 & (90.3) \\
14 & (9.7)\end{aligned}$ & $\begin{array}{l}93(80.9) \\
22(19.1)\end{array}$ & $\begin{array}{l}2.21 \text { (1.08-4.55) } \\
1 \text { (referent) }\end{array}$ & $0.03 *$ & $\begin{array}{l}1.48(0.65-3.35) \\
1 \text { (referent) }\end{array}$ & 0.35 \\
\hline $\begin{array}{l}\text { Information } \\
\text { High } \\
\text { Medium } \\
\text { Low }\end{array}$ & $\begin{array}{l}61(42.1) \\
45(31.0) \\
39(26.9)\end{array}$ & $\begin{array}{l}33(28.7) \\
40(34.8) \\
42(36.5)\end{array}$ & $\begin{array}{l}2.04(1.11-3.76) \\
1.24 \text { (0.67-2.29) } \\
1 \text { (referent) }\end{array}$ & $\begin{array}{l}0.02 * \\
0.49\end{array}$ & $\begin{array}{l}1.99(1.05-3.77) \\
1.11 \text { (0.59-2.10) } \\
1 \text { (referent) }\end{array}$ & $\begin{array}{l}0.04^{*} \\
0.75\end{array}$ \\
\hline
\end{tabular}


Health Bureau, Taipei County, and two interviewers Ms Wan-Jung Yu and Ms Hsin-Lun Li from Taipei College of Nursing.

\section{Authors' affiliations}

Chia-Ming Li, Consultant of Family Medicine (in charge of data collection and analyses), Department of Community and Family Medicine, National University Hospital, Bei-Hu Branch, Taipei, Taiwan Ming-Neng Shiu, (expert in medical screening), Public Health Bureau of Taipei County Government, Taiwan

Shu-Li Chia, Division head of health promotion of Public Health Bureau (in charge of record management and arrangement of telephone interview), Public Health Bureau of Taipei County Government, Taiwan

Jen-Pei Liu Professor of Biostatistics, Division of Biostatistics, Department of Agronomy, National Taiwan University, Taipei, Taiwan Tony Hsiu-Hsi Chen, Professor of Biostatistics and Medical Screening, Institute of Preventive Medicine and Department of Public Health, National Taiwan University, Taipei, Taiwan

Wei-Chu Chie, Professor of Preventive Medicine, Institute of Preventive Medicine and Department of Public Health, National Taiwan University, Taipei, Taiwan

\section{REFERENCES}

1 Taiwan Cancer Registry. Annual report. Incidence of colorectal cancer. 2002; http://crs.cph.ntu.edu.tw/crs_c/annual.html

2 Department of Health, R.O.C (Taiwan). Trend of mortality rate of major cancer in Taiwan 2004 http://www doh gov.tw/statistic/data/

3 Ransohoff DF, Lang CA. Screening for colorectal cancer with the fecal occult blood test: a background paper. Ann Intern Med 1997; 1 26:81 1-22

4 Mandel JS, Bond JH, Church TR, et al. Reducing mortality from colorectal cancer by screening for fecal occult blood. $N$ Engl J Med 1993:328:1365-71

5 Hardcastle JD, Chamberlain JO, Robinson MH, et al. Randomised controlled trial of faecal-occult-blood screening for colorectal cancer. Lancet 1996;348:1472-6

6 Kronborg $\mathrm{O}$, Fenger $\mathrm{C}$, Olsen J, et al. Randomised study of screening for colorectal cancer with faecal-occult-blood test. Lancet 1996;348: 1467-71

7 Etzioni DA, Yano EM, Rubenstein LV, et al. Measuring the quality of colorectal cancer screening: the importance of follow-up. Dis Colon Rectum 2006;49:1002-10

8 Byers T, Levin B, Rothenberger D, et al. American Cancer Society guidelines for screening and surveillance for early detection of colorectal polyps and cancer: update 1997. American Cancer Society Detection and Treatment Advisory Group on Colorectal Cancer. CA Cancer J Clin 1997:47: 154-60

9 US Preventive Services Task Force (USPSTF) Recommendations and Rationale Screening for Colorectal Cancer. http://www.ahrq.gov/clinic/ 3rduspstf/colorectal/colorr.htm

10 Janz NK, Champion VL, Strecher VJ. The Health Belief Model In: Glanz K, Rimer BK, Lewis FM, eds. Health Behavior and Health Education: Theory, Research, and Practice. 3rd edn. San Francisco: Jossey-Bass, 2002;45-65

11 Baig N, Myers RE, Turner BJ, et al. Physician-reported reasons for limited follow-up of patients with a positive fecal occult blood test screening result. Am J Gastroenterol 2003;98:2078-81

12 Shields HM Weiner MS, Henry DR, et al. Factors that influence the decision to do an adequate evaluation of a patient with a positive stool for occult blood. Am J Gastroenterol 2001;96:196-203

13 Marcus AC, Crane LA, Kaplan CP, et al. Improving adherence to screening follow-up among women with abnormal Pap smears. Med Care 1992;30:216-30

14 Fox $\mathrm{P}$, Arnsberger $\mathrm{P}$, Zhang $X$. An examination of differential follow-up rates in cervical cancer screening. J Community Health 1997;22:199-209

15 Melnikow J, Chan BKS, Stewart GK. Do follow-up recommendations for abnormal Papanicolaou smears influence patient adherence? Arch Fam Med 1999:8:510-14

16 Jones BA, Novis DA. Follow-up of abnormal gynecologic cytology. Arch Pathol Lab Med 2000; 1 24:665-71

17 McCarthy BD, Yood MU, Janz NK, et al. Evaluation of factors potentially associated with inadequate follow-up of mammographic abnormalities. Cancer 1996;77:2070-6

18 Pulkki L, Kivimaki M, Keltikangas-Jarrinen L, et al. Contribution of adolescent and early adult personality to the inverse association between education and cardiovascular risk behaviors: prospective populationbased cohort study. Int J Epidemiol 2003:32:968-75

19 Lerman CP, Hanjanan P, Caputo S, et al. Telephone consulting improves adherence to colonoscopy among lower-income minority women. J Clin Oncol 1992;10:330-3

20 Chen YD. The Emotional Reaction and Seeking Medical Service Behavior of Positive Colorectal Cancer Screening Result Subjects. ROC (Taiwan): Research Project of Bureau of Health Promotion, Department of Health, 2005

21 Levin B, Hess K, Johnson C. Screening for colorectal cancer. A comparison of 3 fecal occult blood tests. Arch Intern Med 1997;157:970-6
22 Paskett ED, Carter WB, Chu J, White E Compliance behavior in women with abnormal Pap smears. Developing and testing a decision model. Med Care 1990;28:643-56

23 Weissman JS, Stern R, Fielding SL, et al. Delayed access to health care: risk factors, reasons and consequences. Ann Intern Med 1991;114: 325-331

\section{APPENDIX 1}

\section{Health Belief Model ${ }^{10}$}

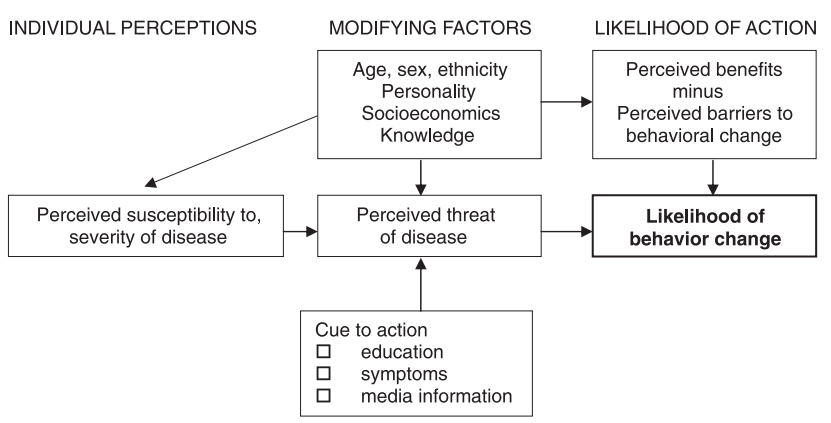

\section{APPENDIX 2}

\section{Questionnaire used in the research}

\section{Answers: for attitudes or beliefs - Likert's scale 1-5; for} facts: multiple choices

Perceived susceptibility

(1) When someone notified you that your faecal occult blood test (FOBT) was abnormal, what did you think about the result?

(2) Have you worried after knowing your FOBT is positive?

(3) 'If there is no discomfort, it is not necessary to have any further medical examinations. ' Do you agree with it?

Severity of disease

Do you agree that 'colon cancer is a severe disease'.

Perceived health status

How do you feel about your health condition?

Casual personality

(1) One knows his or her own body the best, so regular physical examinations are not necessary. Do you agree with the above statement?

(2) No matter what may cause a positive FOBT, do you feel less worried if you would accept further examinations?

(3) Do you usually accept medical professionals' recommendations?

Knowledge

(1) Do you know the purpose of FOBT?

(2) Do you know what medical procedures should be performed after diagnosing a positive FOBT?

(3) Do you know any other reasons besides haemorrhoids that may cause a positive FOBT?

Cue to action - symptom

(1) Have you had any anal bleeding when moving your bowel in the past one year?

(2) Have you sometimes felt abdominal discomfort in the recent one year? 
Cue to action - family's worry

(1) My health is important for my family. Do you agree with the above statement?

(2) Will your families worry about you if you do not accept colon examinations?

Cue to action - information (including media, education)

(1) Did the health centre staff clearly explain the meaning of a positive FOBT to you?

(2) Have you ever learned the information that people with a positive FOBT should have further examinations from TV, radio or newspaper?

(3) Have your relatives or friends ever told you their experiences about colonoscopy?

(4) Have your relatives or friends ever had colon cancer or polyp?

(5) Did your relatives or friends ever encourage you to accept further examinations after you knew you had a positive FOBT?

(6) Have you ever asked the god's approval for having further examinations after learning a positive FOBT?

(7) Have any medical professional ever encouraged you to have further examinations after you had a positive FOBT?

(8) Did any medical professional ever clearly explain the reasons for having colonoscopy?

Perceived benefits

(1) Do you agree that 'FOBT may detect colon cancer early'.
(2) Do you agree that 'the earlier the colon cancer could be diagnosed, the better the treatment result would be'?

(3) Do you agree that 'colonoscopy or double-contrast lower gastrointestinal examination can detect colon cancer in the early stage'.

Barriers to behaviour change

(1) Do you think it is too time consuming to have a colonoscopy?

(2) After having a positive FOBT, did your colonoscopy have an inconvenient or conflicting schedule?

(3) Do you think colonoscopy is uncomfortable?

(4) Will you feel nervous about colonoscopy?

(5) Can you afford colonoscopy?

\section{Fixed health provider}

When you had any health problems, did you usually visit your fixed primary physician?

Personal health practice

(1) Have you smoked in the past one year?

(2) Have you drunk alcohol in the past one year?

(3) Have you chewed beetle nut in the past one year?

(4) Do you eat at least five kinds of vegetables and fruits everyday?

(5) Do you exercise at least three times every week? 